\title{
A Perceptual Model for Sinusoidal Audio Coding Based on Spectral Integration
}

\author{
Steven van de Par \\ Digital Signal Processing Group, Philips Research Laboratories, 5656 AA Eindhoven, The Netherlands \\ Email: steven.van.de.par@philips.com \\ Armin Kohlrausch \\ Digital Signal Processing Group, Philips Research Laboratories, 5656 AA Eindhoven, The Netherlands \\ Department of Technology Management, Eindhoven University of Technology, 5600 MB Eindhoven, The Netherlands \\ Email: armin.kohlrausch@philips.com
}

\section{Richard Heusdens}

Department of Mediamatics, Delft University of Technology, 2600 GA Delft, The Netherlands Email: r.heusdens@ewi.tudelft.nl

\author{
Jesper Jensen \\ Department of Mediamatics, Delft University of Technology, 2600 GA Delft, The Netherlands \\ Email: j.jensen@ewi.tudelft.nl
}

\section{Søren Holdt Jensen}

Department of Communication Technology, Institute of Electronic Systems, Aalborg University, DK-9220 Aalborg, Denmark Email:shj@kom.aau.dk

\section{Received 31 October 2003; Revised 22 July 2004}

Psychoacoustical models have been used extensively within audio coding applications over the past decades. Recently, parametric coding techniques have been applied to general audio and this has created the need for a psychoacoustical model that is specifically suited for sinusoidal modelling of audio signals. In this paper, we present a new perceptual model that predicts masked thresholds for sinusoidal distortions. The model relies on signal detection theory and incorporates more recent insights about spectral and temporal integration in auditory masking. As a consequence, the model is able to predict the distortion detectability. In fact, the distortion detectability defines a (perceptually relevant) norm on the underlying signal space which is beneficial for optimisation algorithms such as rate-distortion optimisation or linear predictive coding. We evaluate the merits of the model by combining it with a sinusoidal extraction method and compare the results with those obtained with the ISO MPEG-1 Layer I-II recommended model. Listening tests show a clear preference for the new model. More specifically, the model presented here leads to a reduction of more than $20 \%$ in terms of number of sinusoids needed to represent signals at a given quality level.

Keywords and phrases: audio coding, psychoacoustical modelling, auditory masking, spectral masking, sinusoidal modelling, psychoacoustical matching pursuit.

\section{INTRODUCTION}

The ever-increasing growth of application areas such as consumer electronics, broadcasting (digital radio and television), and multimedia/Internet has created a demand for

This is an open-access article distributed under the Creative Commons Attribution License, which permits unrestricted use, distribution, and reproduction in any medium, provided the original work is properly cited. high-quality digital audio at low bit rates. Over the last decade, this has led to the development of new coding techniques based on models of human auditory perception (psychoacoustical masking models). Examples include the coding techniques used in the ISO/IEC MPEG family, for example, [1], the MiniDisc from Sony [2], and the digital compact cassette (DCC) from Philips [3]. For an overview of recently proposed perceptual audio coding schemes and standards, we refer to the tutorial paper by Painter and Spanias [4]. 
A promising approach to achieve low bit rate coding of digital audio signals with minimum perceived loss of quality is to use perception-based hybrid coding schemes, where audio signals are decomposed and coded as a sinusoidal part and a residual. In these coding schemes, different signal components occurring simultaneously are encoded with different encoders. Usually, tonal components are encoded with a specific encoder aimed at signals composed of sinusoids and the remaining signal components are coded with a waveform or noise encoder $[5,6,7,8,9]$. To enable the selection of the perceptually most suitable sinusoidal description of an audio signal, dedicated psychoacoustical models are needed and this will be the topic of this paper.

One important principle by which auditory perception can be exploited in general audio coding is that the modelling error generated by the audio coding algorithm is masked by the original signal. When the error signal is masked, the modified audio signal generated by the audio coding algorithm is indistinguishable from the original signal.

To determine what level of distortion signal is allowable, an auditory masking model can be used. We, for example, consider the case where the masking model is used in a transform coder. Here the model will specify, for each spectrotemporal interval within the original audio signal, what distortion level can be allowed within that interval such that it is perceptually just not detectable. With an appropriate signal transformation, for example, an MDCT filter bank [10, 11], it is possible to selectively adapt the accuracy with which each different spectro-temporal interval is described, that is, the number of bits used for quantisation. In this way, the spectro-temporal characteristics of the error signal, can be adapted such that auditory masking is exploited effectively, leading to the lowest possible bit rate without perceptible distortions.

Most existing auditory masking models are based on the psychoacoustical literature that predominantly studied the masking of tones by noise signals (e.g., [12]). Interestingly, for subband coders and transform coders the nature of the signals is just the reverse; the distortion is noise-like, while the masker, or original signal, is often tonal in character. Nevertheless, based on this psychoacoustical literature dedicated psychoacoustical models have been developed for audio coding for the situation where the distortion signal is noise-like such as the ISO MPEG model [1].

Masking models are also used for sinusoidal coding, where the signal is modelled by a sum of sinusoidal components. Most existing sinusoidal audio coders, for example, $[5,6,13]$ rely on masking curves derived from spectralspreading-based perceptual models in order to decide which components are masked by the original signal, and which are not. As a consequence of this decision process, a number of masked components are rejected by the coder, resulting in a distortion signal that is sinusoidal in nature. In this paper a model is introduced that is specifically designed for predicting the masking of sinusoidal components. In addition, the proposed model takes into account some new findings in the psychoacoustical literature about spectral and temporal integration in auditory masking.
This paper is organised as follows. In Section 2 we discuss the psychoacoustical background of the proposed model. Next, in Section 3, the new psychoacoustical model will be introduced, followed by Section 4, which describes the calibration of the model. Section 5 compares predictions of the model with some basic psychoacoustical findings. In Section 6, we apply the proposed model in a sinusoidal audio modelling method and in Section 7 we compare, in a listening test, the resulting audio quality to that obtained with the ISO MPEG model [1]. Finally, in Section 8, we will present some conclusions.

\section{PSYCHOACOUSTICAL BACKGROUND}

Auditory masking models that are used in audio coding are predominantly based on a phenomenon known as simultaneous masking (see, e.g., [14]). One of the earlier relevant studies goes back to Fletcher [15] who performed listening experiments with tones that were masked by noise. In his experiments the listeners had to detect a tone that was presented simultaneously with a bandpass noise masker that was spectrally centred around the tone. The threshold level for detecting the tones was measured as a function of the masker bandwidth while the power spectral density (spectrum level) was kept constant. Results showed that an increase of bandwidth, thus increasing the total masker power, led to an increase of the detection thresholds. However, this increase was only observed when the bandwidth was below a certain critical bandwidth; beyond this critical bandwidth, thresholds were independent of bandwidth. These observations led to the critical band concept which is the spectral interval across which masker power is integrated to contribute to the masking of a tone centred within the interval.

An explanation for these observations is that the signal processing in the peripheral auditory system, specifically by the basilar membrane in the cochlea, can be represented as a series of bandpass filters which are excited by the input signal, and which produce parallel bandpass-filtered outputs (see, e.g., [16]). The detection of the tone is thought to be governed by the bandpass filter (or auditory filter) that is centred around the tone. When the power ratio between the tone and the masker at the output of this filter exceeds a certain criterion value, the tone is assumed to be detectable. With these assumptions the observations of Fletcher can be explained; as long as the masker has a bandwidth smaller than that of the auditory filter, an increase in bandwidth will also lead to an increase in the masker power seen at the output of the auditory filter, which, in turn, leads to an increase in detection threshold. Beyond the auditory filter bandwidth the added masker components will not contribute to the masker power at the output of the auditory filter because they are rejected by the bandpass characteristic of the auditory filter. Whereas in Fletchers experiments the tone was centred within the noise masker, later on experiments were conducted where the masker did not spectrally overlap with the tone to be detected (see, e.g., [17]). Such experiments reveal more information on the auditory filter characteristic, specifically about the tails of the filters. 
The implication of such experiments should be treated with care. When different maskers and signals are chosen, the resulting conclusions about the auditory filter shape are quite different. For example, a tonal masker proves to be a much poorer masker than a noise signal [17]. In addition, the filter shapes seem to depend on the masker type as well as on the masker level. These observations suggest that the basic assumptions of linear, that is, level independent, auditory filters and an energy criterion that defines audibility of distortion components, are only a first-order approximation and that other factors play a role in masking. For instance, it is known that the basilar membrane behaves nonlinearly [18], which may explain, for instance, the level dependence of the auditory filter shape. For a more elaborate discussion of auditory masking and auditory filters, the reader is referred to $[19,20,21]$.

Despite the fact that the assumption of a linear auditory filter and an energy detector can only be regarded as a firstorder approximation of the actual processing in the auditory system, we will proceed with this assumption because it proves to give very satisfactory results in the context of audio coding with relatively simple means in terms of computational complexity.

Along similar lines as outlined above, the ISO MPEG model [1] assumes that the distortion or noise level that is allowed within a specific critical band is determined by the weighted power addition of all masker components spread on and around the critical band containing the distortion. The shape of the weighting function that is applied is based on auditory masking data and essentially reflects the underlying auditory filter properties. These "spectral-spreading"based perceptual models have been used in various parametric coding schemes for sinusoidal component selection $[5,6,13]$. It should be noted that in these models, it is assumed that only the auditory filter centred around the distortion determines the detectability of the distortion. When the distortion-to-masker ratio is below a predefined threshold value in each auditory filter, the distortion is assumed to be inaudible. On the other hand, when one single filter exceeds this threshold value, the distortion is assumed to be audible. This assumption is not in line with more recent insights in the psychoacoustical literature on masking and will later in the paper be shown to have a considerable impact on the predicted masking curves. Moreover, in the ISO MPEG model [1], a distinction is made between masking by noisy and tonal spectral components to be able to account for the difference in masking power of these signal types. For this purpose a tonality detector is required which, in the Layer I model, is based on a spectral peak detector.

Threshold measurements in psychoacoustical literature consistently show that a detection threshold is not a rigid threshold. A rigid threshold would imply that if the signal to be detected would be just above the detection threshold, the signal would always be detected while it would never be detected when it would be just below the threshold. Contrary to this pattern, it is observed in detection threshold measurements that the percentages of correct detection as a function of signal level follow a sigmoid psychometric function [22].
The detection threshold is defined as the level for which the signal is detected correctly with a certain probability of, typically, $70 \%-75 \%$.

In various theoretical considerations, the shape of the psychometric function is explained by assuming that within the auditory system some variable, for example, the stimulus power at the output of an auditory filter, is observed. In addition, it is assumed that noise is present in this observation due to, for example, internal noise in the auditory system. When the internal noise is assumed to be Gaussian and additive, the shape of the sigmoid function can be predicted. For the case that a tone has to be detected within broadband noise, the assumption of a stimulus power measurement with additive Gaussian noise leads to good predictions of the psychometric function. When the increase in the stimulus power caused by the presence of the tonal signal is large compared to the standard deviation of the internal noise, high percentages of correct detection are expected while the reverse is true for small increases in stimulus power. The ratio between the increase in stimulus power and the standard deviation of the internal noise is defined as the sensitivity index $d^{\prime}$ and can be calculated from the percentage of correct responses of the subjects. This theoretical framework is based on signal detection theory and is described more extensively in, for example, [23].

In several more recent studies it is shown that the audibility of distortion components is not determined solely by the critical band with the largest audible distortion [24, 25]. Buus et al. [24] performed listening tests where tone complexes had to be detected when presented in a noise masker. They first measured the threshold levels of several tones separately each of which were presented simultaneously with wideband noise. Due to the specific spectral shape of the masking noise, thresholds for individual tones were found to be constant across frequency. In addition to the threshold measurements for a single tone, thresholds were also measured for a complex of 18 equal-level tones. The frequency spacing of the tones was such that each auditory critical band contained only a single tone. If the detectability of the tones was only determined by the filter with the best detectable tone, the complex of tones would be just audible when one individual component of the complex had the same level as the measured threshold level of the individual tones. However, the experiments showed that thresholds for the tone complex were considerably lower than expected based on the bestfilter assumption, indicating that information is integrated across auditory filters.

In the paper by Buus et al. [24], a number of theoretical explanations are presented. We will discuss only the multiband detector model [23]. This model assumes that the changes in signal power at the output of each auditory filter are degraded by additive internal noise that is independent in each auditory filter. It is then assumed that an optimally weighted sum of the signal powers at the outputs of the various auditory filters is computed which serves as a new decision variable. Based on these assumptions, it can be shown that the sensitivity index of a tone complex, 
$d_{\text {total }}^{\prime}$, can be derived from the individual sensitivity indices $d_{n}^{\prime}$ as follows:

$$
d_{\text {total }}^{\prime}=\sqrt{\sum_{n=1}^{K} d_{n}^{\prime 2}},
$$

where $K$ denotes the number of tones and where each individual sensitivity index is proportional to the tone-to-masker power ratio [22]. According to such a framework, each doubling of the number of auditory filters that can contribute to the detection process will lead to a reduction of $1.5 \mathrm{~dB}$ in threshold. The measured thresholds by Buus et al. are well in line with this prediction. In their experiments, the complex of 18 tones leads to a reduction of $6 \mathrm{~dB}$ in detection threshold as compared to the detection threshold of a single tone. Based on (1) a change of $6.3 \mathrm{~dB}$ was expected. More recently, Langhans and Kohlrausch [25] performed similar experiments with complex tones having a constant spacing of $10 \mathrm{~Hz}$ presented in a broadband noise masker, confirming that information is integrated across auditory filters. In addition, results obtained by van de Par et al. [26] indicate that also for bandpass noise signals that had to be detected against the background of wideband noise maskers, the same integration across auditory filters is observed.

As indicated, integration of information across a wide range of frequencies is found in auditory masking. Similarly, integration across time has been shown to occur in the auditory system. Van den Brink [27] investigated the detection of tones of variable duration that were presented simultaneously with a noise masker with a fixed duration that was always longer than that of the tone. Increasing the duration of the tone reduced the detection thresholds up to a duration of about 300 milliseconds. While this result is an indication of integration across time, it also shows that there is a limitation in the interval for which temporal integration occurs.

The above findings with respect to spectral and temporal integration of information in auditory masking have implications for audio coding which have not been considered in previous studies. On the one hand it influences the masking properties of complex signals as will be discussed in Section 5, on the other hand it has implications for rate distortion optimisation algorithms. To understand this, consider the case where for one particular frequency region a threshold level is determined for distortions that can be introduced by an audio coder. For another frequency region a threshold can be determined similarly. When both distortions are presented at the same time, the total distortion is expected to become audible due to the spectral integration given by (1). This is in contrast to the more conventional models, such as the ISO MPEG model [1], which would predict this simultaneous distortion to be inaudible.

The effect of spectral integration, of course, can easily be compensated for by reducing the level of the masking thresholds such that the total distortion will be inaudible. But, based on (1), assuming that it holds for masking by complex audio signals, there are many different solutions to this equation which lead to the same $d_{\text {total }}^{\prime}$. In other words, many

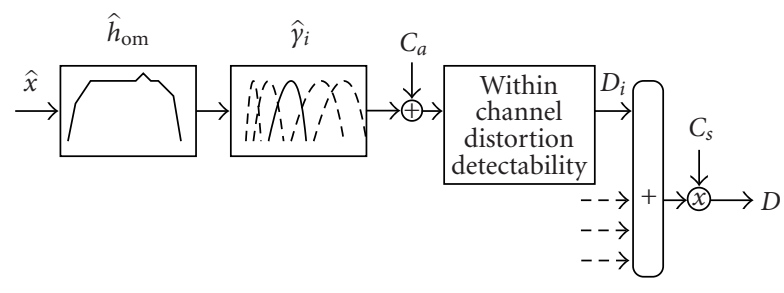

Figure 1: Block diagram of the masking model.

different distributions of distortion levels per spectral region will lead to the same total sensitivity index. However, not every distribution of distortion levels will lead to the same amount of bits spent by the audio coder. Thus, the concept of a masking curve which determines the maximum level of distortion allowed within each frequency region is too restrictive and can be expected to lead to suboptimal audio coders. In fact, spectral distortion can be shaped such that the associated bit rate is minimised. For more information the reader is referred to a study where these ideas were confirmed by listening tests [28].

\section{DESCRIPTION OF THE MODEL}

In line with various state-of-the-art auditory models that have been presented in the psychoacoustical literature, for example, [29], the structure of the proposed model follows the various stages of auditory signal processing. In view of the computational complexity, the model is based on frequency domain processing and consequently neglects some parts of peripheral processing, such as the hair cell transformation which performs inherent nonlinear time-domain processing.

A block diagram of the model is given in Figure 1. The model input $\hat{x}$ is the frequency domain representation of a short windowed segment of audio. The window should lead to sufficient rejection of spectral side lobes in order to facilitate adequate spectral resolution of the auditory filters. The first stage of the model resembles the outer- and middleear transfer function $\hat{h}_{\text {om }}$, which is related to the filtering of the ear canal and the ossicles in the middle ear. The transfer function is chosen to be the inverse of the threshold-in-quiet function $\hat{h}_{\mathrm{tq}}$. This particular shape is chosen to obtain an accurate prediction of the threshold-in-quiet function when no masker signal is present.

The outer- and middle-ear transfer function is followed by a gammatone filter bank (see, e.g., [30]) which resembles the filtering property of the basilar membrane in the inner ear. The transfer function of an $n$ th-order gammatone filter has a magnitude spectrum that is approximated well by

$$
\hat{\gamma}(f)=\left(1+\left(\frac{f-f_{0}}{k \operatorname{ERB}\left(f_{0}\right)}\right)^{2}\right)^{-n / 2},
$$

where $f_{0}$ is the centre frequency of the filter, $\operatorname{ERB}\left(f_{0}\right)$ is the equivalent rectangular bandwidth of the auditory filter centred at $f_{0}$ as suggested by Glasberg and Moore [31], $n$ is 
the filter order which is commonly assumed to be 4 , and $k=2^{(n-1)}(n-1) ! / \pi(2 n-3) ! !$, a factor needed to ensure that the filter indeed has the specified ERB. The centre frequencies of the filters are uniformly spaced on an ERB-rate scale and follow the bandwidths as specified by the ERB scale [31]. The power at the output of each auditory filter is measured and a constant $C_{a}$ is added to this output as a means to limit the detectability of very weak signals at or below the threshold in quiet.

In the next stage, within-channel distortion detectabilities are computed and are defined as the ratios between the distortion and the masker-plus-internal noise seen at the output of each auditory filter. In fact, the within-channel distortion detectability $D_{i}$ is proportional to the sensitivity in$\operatorname{dex} d^{\prime}$ as described earlier. This is an important step; the distortion detectability ( or $d^{\prime}$ ) will be used as a measure of perceptual distortion. This perceptual distortion measure can be interpreted as a measure of the probability that subjects can detect a distortion signal in the presence of a masking signal. The masker power within the $i$ th filter due to an original (masking) signal $x$ is given by

$$
M_{i}=\frac{1}{N} \sum_{f}\left|\hat{h}_{\mathrm{om}}(f)\right|^{2}\left|\hat{\gamma}_{i}(f)\right|^{2}|\hat{x}(f)|^{2}
$$

where $N$ is the segment size in number of samples. Equivalently, the distortion power within the $i$ th filter due to a distortion signal $\varepsilon$ is given by

$$
S_{i}=\frac{1}{N} \sum_{f}\left|\hat{h}_{\mathrm{om}}(f)\right|^{2}\left|\hat{\gamma}_{i}(f)\right|^{2}|\widehat{\varepsilon}(f)|^{2} .
$$

Note that $(1 / N)|\hat{x}(f)|^{2}$ denotes the power spectral density of the original, masking signal in sound pressure level (SPL) per frequency bin, and similarly $(1 / N)|\widehat{\varepsilon}(f)|^{2}$ is the power spectral density of the distorting signal. The within-channel distortion detectability $D_{i}$ is given by

$$
D_{i}=\frac{S_{i}}{M_{i}+(1 / N) C_{a}}
$$

From this equation two properties of the within-channel distortion detectability $D_{i}$ can be seen. When the distortion-tomasker ratio $S_{i} / M_{i}$ is kept constant while the masker power is much larger than $(1 / N) C_{a}$, distortion detectability is also constant. In other words, at medium and high masker levels the detectability $D_{i}$ is mainly determined by the distortionto-masker ratio. Secondly, when the masker power is small compared to $(1 / N) C_{a}$, the distortion detectability is independent of the masker power, which resembles the perception of signals near the threshold in quiet.

In line with the multiband energy detector model [23], we assume that within-channel distortion detectabilities $D_{i}$ are combined into a total distortion detectability by an additive operation. However, we do not add the squared sensitivity indices as in (1), but we simply add the indices directly. Although this may introduce inaccuracies, these will later turn out to be small. A benefit of this choice is that the distortion measure that will be derived from this assumption will have properties that allow a computationally simple formulation of the model (see (11)). In addition, recent results [26] show that at least for the detection of closely spaced tones $(20 \mathrm{~Hz}$ spacing) masked by noise, the reduction in thresholds when increasing the signal bandwidth is more in line with a direct addition of distortion detectabilities than with (1). Therefore, we state that

$$
\begin{aligned}
D(x, \varepsilon) & =C_{s} L_{\text {eff }} \sum_{i} D_{i} \\
& =C_{s} L_{\text {eff }} \sum_{i} \frac{\sum_{f}\left|\hat{h}_{\mathrm{om}}(f)\right|^{2}\left|\hat{\gamma}_{i}(f)\right|^{2}|\widehat{\varepsilon}(f)|^{2}}{N M_{i}+C_{a}},
\end{aligned}
$$

where $D(x, \varepsilon)$ is the total distortion detectability as it is predicted for a human observer given an original signal $x$ and a distortion signal $\varepsilon$. The calibration constant $C_{s}$ is chosen such that $D=1$ at the threshold of detectability. To account for the dependency of distortion detectability on the duration of the distortion signal (in line with [27]), a scaling factor $L_{\text {eff }}$ is introduced defined as

$$
L_{\mathrm{eff}}=\min \left(\frac{L}{300 \mathrm{~ms}}, 1\right)
$$

where $L$ is the segment duration in milliseconds. Equation (8) resembles the temporal integration time of the human auditory system which has an upper bound of 300 milliseconds [27]. ${ }^{1}$

Equation (7) gives a complete description of the model. However, it defines only a perceptual distortion measure and not a masking curve such as is widely used in audio coding nor a masked threshold such as is often used in psychoacoustical experiments.

In order to derive a masked threshold, we assume that the distortion signal $\hat{\varepsilon}(f)=A \hat{\epsilon}$. Here, $A$ is the amplitude of the distortion signal and $\hat{\epsilon}$ the normalised spectrum of the distortion signal such that $\|\epsilon\|^{2}=1$ which is assumed to correspond to a sound pressure level of $0 \mathrm{~dB}$. Without yet making an assumption about the spectral shape of $\epsilon$, we can derive that, assuming that $D=1$ at the threshold of detectability, the masked threshold $A^{2}$ for the distortion signal $\epsilon$ is given by

$$
\frac{1}{A^{2}}=C_{s} L_{\mathrm{eff}} \sum_{i} \frac{\sum_{f}\left|\hat{h}_{\mathrm{om}}(f)\right|^{2}\left|\hat{\gamma}_{i}(f)\right|^{2}|\hat{\epsilon}(f)|^{2}}{N M_{i}+C_{a}}
$$

When deriving a masking curve it is important to consider exactly what type of signal is masked. When a masking model is used in the context of a waveform coder, the

\footnotetext{
${ }^{1}$ An alternative definition would be to state that $L_{\text {eff }}=N$, the total duration of the segment in number of samples. According to this definition it is assumed that distortions are integrated over the complete excerpt at hand, which is not in line with perceptual masking data, but which in our experience still leads to very satisfactory results [32].
} 
distortion signal introduced by the coder is typically assumed to consist of bands of noise. For a sinusoidal coder, however, the distortion signal contains the sinusoids that are rejected by the perceptual model. Thus, the components of the distortion signal are in fact more sinusoidal in nature. Assuming now that a distortion component is present in only one bin of the spectrum, we can derive the masked thresholds for sinusoidal distortions. We assume that $\hat{\epsilon}(f)=\hat{v}\left(f_{m}\right) \delta\left(f-f_{m}\right)$ with $\hat{v}\left(f_{m}\right)$ being the sinusoidal amplitude and $f_{m}$ the sinusoidal frequency. Together with the assumption that $D=1$ at the threshold of detectability, $\hat{v}$ can be derived such that the distortion is just not detectable. In this way, by varying $f_{m}$ over the entire frequency range, $\widehat{v}^{2}$ constitutes the masking curve for sinusoidal distortions in the presence of a masker $x$. By substituting the above assumptions in (7) we obtain

$$
\frac{1}{\hat{v}^{2}\left(f_{m}\right)}=C_{s} L_{\text {eff }} \sum_{i} \frac{\left|\hat{h}_{\mathrm{om}}\left(f_{m}\right)\right|^{2}\left|\hat{\gamma}_{i}\left(f_{m}\right)\right|^{2}}{N M_{i}+C_{a}} \text {. }
$$

Substituting (10) in (7), we get

$$
D(x, \varepsilon)=\sum_{f} \frac{|\widehat{\varepsilon}(f)|^{2}}{\hat{v}^{2}(f)} .
$$

This expression shows that the computational load for calculating the perceptual distortion $D(x, \varepsilon)$ can be very low once the masking curve $\hat{v}^{2}$ has been calculated. This simple form of the perceptual distortion, such as given in (11), arises due to the specific choice of the addition as defined in (6).

\section{CALIBRATION OF THE MODEL}

For the purpose of calibration of the model, the constants $C_{a}$ for absolute thresholds and $C_{s}$ for the general sensitivity of the model in (7) need to be determined. This will be done using two basic findings from the psychoacoustical literature, namely the threshold in quiet and the just noticeable difference (JND) in level of about $0.5-1 \mathrm{~dB}$ for sinusoidal signals [33].

When considering the threshold in quiet, we assume that the masking signal is equal to zero, that is, $\hat{x}=0$ and that the just detectable sinusoidal distortion signal is given by $\hat{\varepsilon}(f)=\hat{h}_{\mathrm{tq}}\left(f_{m}\right) \delta\left(f-f_{m}\right)$ for some $f_{m}$, where $\hat{h}_{\mathrm{tq}}$ is the threshold-in-quiet curve. By substituting these assumptions in (7) (assuming that $D=1$ corresponds to a just detectable distortion signal), we obtain

$$
C_{a}=C_{s} L_{\text {eff }} \sum_{i}\left|\hat{\gamma}_{i}\left(f_{m}\right)\right|^{2}
$$

Note that (12) only holds if $\sum_{i}\left|\hat{\gamma}_{i}\left(f_{m}\right)\right|^{2}$ is constant for all $f_{m}$, which is approximately true for gammatone filters.

We assume a $1 \mathrm{~dB}$ JND which corresponds to a masking condition where a sinusoidal distortion is just detectable in the presence of a sinusoidal masker at the same frequency, say $f_{m}$. For this to be the case, the distortion level has to be $18 \mathrm{~dB}$ lower than the masker level, assuming that the masker and distortion are added inphase. This specific phase assumption is made because it leads to similar thresholds as when the masker and signal are slightly off-frequency with respect to one another, the case which is most likely to occur in audio coding contexts. We therefore assume that the masker signal is $\hat{x}(f)=A_{70} \delta\left(f-f_{m}\right)$ and the distortion signal $\hat{\mathcal{\varepsilon}}(f)=A_{52} \delta\left(f-f_{m}\right)$, with $A_{70}$ and $A_{52}$ being the amplitudes for a 70 and $52 \mathrm{~dB}$ SPL sinusoidal signal, respectively. Using (3) and (7), this leads to the expression

$$
\frac{1}{C_{s}}=L_{\text {eff }} \sum_{i} \frac{\left|\hat{h}_{\mathrm{om}}\left(f_{m}\right)\right|^{2}\left|\hat{\gamma}_{i}\left(f_{m}\right)\right|^{2} A_{52}^{2}}{\left|\hat{h}_{\mathrm{om}}\left(f_{m}\right)\right|^{2}\left|\hat{\gamma}_{i}\left(f_{m}\right)\right|^{2} A_{70}^{2}+C_{a}} .
$$

When (12) is substituted into (13), an expression is obtained where $C_{s}$ is the only unknown. A numerical solution to this equation can be found using, for example, the bisection method (cf. [34]). A suitable choice for $f_{m}$ would be $f_{m}=1 \mathrm{kHz}$, since it is in the middle of the auditory range. This calibration at $1 \mathrm{kHz}$ does not significantly reduce the accuracy of the model at other frequencies. On the one hand the incorporation of a threshold-in-quiet curve prefilter provides the proper frequency dependence of thresholds in quiet. On the other hand, JNDs do not differ much across frequency both in the model predictions and humans.

\section{MODEL EVALUATION AND COMPARISON WITH PSYCHOACOUSTICAL DATA}

To show the validity of the model, some basic psychoacoustical data from listening experiments will be compared to model predictions. We will consider two cases, namely sinusoids masked by noise and sinusoids masked by sinusoids.

Masking of sinusoids has been measured in several experiments for both (white) noise maskers [12, 35] and for sinusoidal maskers [36]. Figure 2 a shows masking curves predicted by the model for a white noise masker with a spectrum level of $30 \mathrm{~dB} / \mathrm{Hz}$ for a long duration signal (solid line) and a 200 millisecond signal (dashed line) with corresponding listening test data represented by circles [12] and asterisks [35], respectively. Figure $2 \mathrm{~b}$ shows the predicted masking curve (solid line) for a $1 \mathrm{kHz} 50 \mathrm{~dB}$ SPL sinusoidal masker along with corresponding measured masking data [36]. The model predictions are well in line with data for both sinusoidal and noise maskers, despite the fact that no tonality detector was included in the model such as is conventionally needed in masking models for audio coding (e.g., [1]). Only at lower frequencies, there is a discrepancy between the data for the noise masker and the predictions by the model. The reason for this discrepancy may be that in psychoacoustical studies, running noise generators are used to generate the masker signal rather than a single noise realisation, as it is done in audio coding applications. The latter case has, according to several studies, a lower masking strength [37]. This difference in masking strength is due to the inherent masker power fluctuations when a running noise is presented, which depends inversely on the product of time and bandwidth seen at the output of an auditory filter. The narrower the auditory filter (i.e., the lower its centre frequency), the larger these fluctuations will be and the larger the difference is expected to be. 


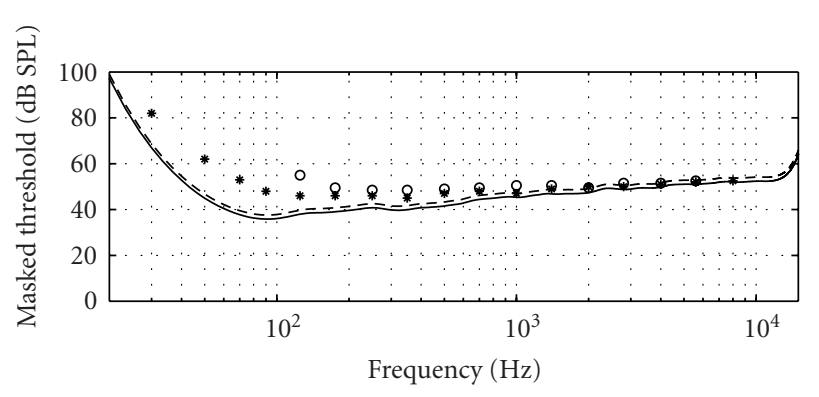

(a)

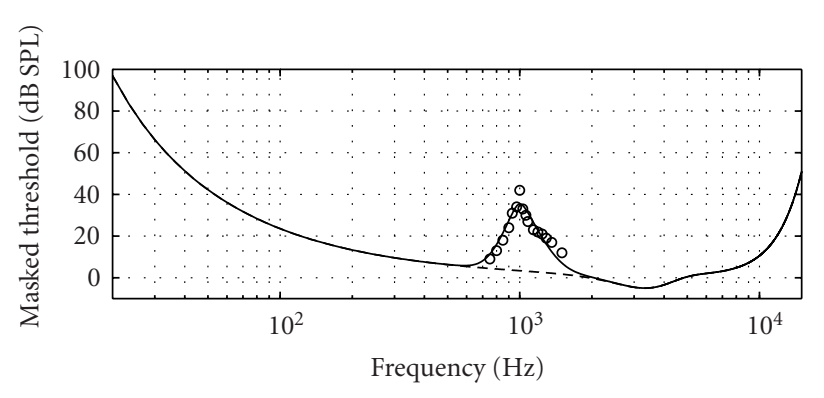

(b)

FIGURE 2: (a) Masking curves predicted by the model for a white noise masker with a spectrum level of $30 \mathrm{~dB} / \mathrm{Hz}$ for a long duration signal (solid line) and a 200-millisecond signal (dashed line) with corresponding listening test data represented by the circles [12] and asterisks [35], respectively. (b) Masking curves for a $1 \mathrm{kHz}$ $50 \mathrm{~dB}$ SPL sinusoidal masker. The dashed line is the threshold in quiet. Circles show data from [36].

As can be seen in Figure 2, the relatively weaker masking power of a sinusoidal signal is predicted well by the model without the need for explicit assumptions about the tonality of the masker such as those included in, for example, the ISO MPEG model [1]. Indeed, in the case of a noise masker (Figure $2 \mathrm{a}$ ), the masker power within the critical band centred around $1 \mathrm{kHz}$ (bandwidth $132 \mathrm{~Hz}$ ) is approximately $51.2 \mathrm{~dB}$ SPL, whereas the sinusoidal masker (Figure 2b) has a power of $50 \mathrm{~dB}$ SPL. Nevertheless, predicted detection thresholds are considerably lower for the sinusoidal masker ( $35 \mathrm{~dB}$ SPL) than for the noise masker ( $45 \mathrm{~dB}$ SPL). The reason why the model is able to predict these data well is that for the tonal masker, the distortionto-masker ratio is constant over a wide range of auditory filters. Due to the addition of within-channel distortion detectabilities, the total distortion detectability will be relatively large. In contrast, for a noise masker, only the filter centred on the distortion component will contribute to the total distortion detectability because the off-frequency filters have a very low distortion-to-masker ratio. Therefore, the wideband noise masker will have stronger masking effect. Note that for narrowband noise signals, the predicted masking power, in line with the argumentation for a sinusoidal masker, will also be weak. This, however, seems to be too conservative [38].

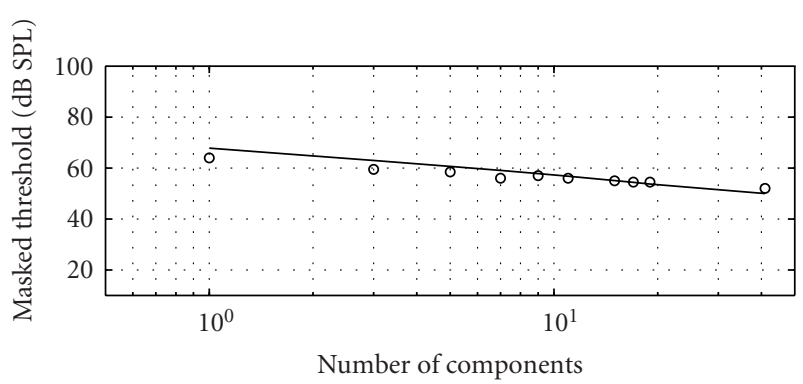

FIGURE 3: Masked thresholds predicted by the model (solid line) and psychoacoustical data (circles) [25]. Masked thresholds are expressed in $\mathrm{dB}$ SPL per component.

A specific assumption in this model is the integration of distortion detectabilities over a wide range of auditory filters. This should allow the model to predict correctly the threshold difference between narrowband distortion signals and more wideband distortion signals. For this purpose an experiment is considered where a complex of tones had to be detected in the presence of masking noise [25]. The tone complex consisted of equal-level sinusoidal components with a frequency spacing of $10 \mathrm{~Hz}$ centred around $400 \mathrm{~Hz}$. The masker was a $0-2 \mathrm{kHz}$ noise signal with an overall level of $80 \mathrm{~dB}$ SPL. The number of components in the complex was varied from one up to 41 . The latter case corresponds to a bandwidth of $400 \mathrm{~Hz}$, which implies that the tone complex covers more than one critical band. Equation (9) was used to derive masked thresholds. As can be seen in Figure 3, there is a good correspondence between the model predictions and the data from [25]. Therefore, it seems that the choice of the linear addition that was made in (6) did not lead to large discrepancies between psychoacoustical data and model predictions.

To conclude this section, a comparison is made between predictions of the MPEG-1 Layer I [1] and the model presented in this study which incorporates spectral integration in masking. The MPEG model is one of a family of models used in audio coding that are based on spectral-spreading functions to model spectral masking. When the masking of a narrowband distortion signal is considered, it is assumed that the auditory filter that is spectrally centred on this distortion signal determines whether the distortion is audible or not. When the energy ratio between distortion signal and masking signal as seen at the output of this auditory filter is smaller than a certain criterion value, the distortion is inaudible. In this manner the maximum allowable distortion signal level at each frequency can be determined which constitutes the masking curve. An efficient implementation for calculating this masking curve is a convolution between the masker spectrum and a spreading function both represented on a Bark scale. The Bark scale is a perceptually motivated frequency scale similar to the ERB-rate scale [39].

The spectral integration model presented here does not consider only a single auditory filter to contribute to the detection of distortions, but potentially a whole range of 


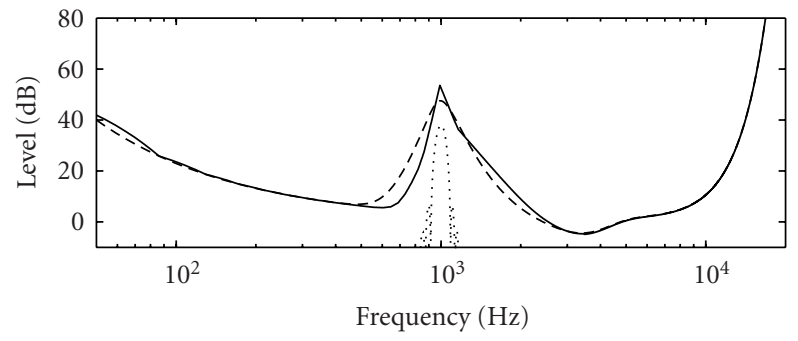

(a)

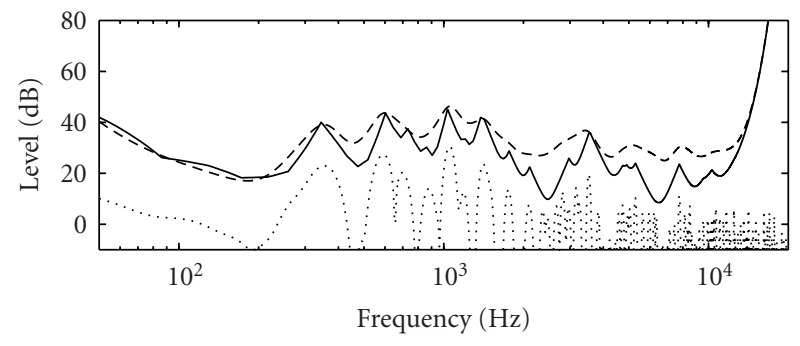

(b)

Figure 4: Masked thresholds predicted by the spectral integration model (dashed line) and the ISO MPEG model (solid line). The masking spectrum (dotted line) is for (a) a $1 \mathrm{kHz}$ sinusoidal signal and (b) a short segment of a harpsichord signal.

filters. This can have a strong impact on the predicted masking curves. Figure $4 \mathrm{a}$ shows the masking curves for a sinusoidal masker at $1 \mathrm{kHz}$ for the MPEG model (solid line) and the spectral integration model (dashed line). The spectrum of the sinusoidal signal is also plotted (dotted line), but scaled down for visual clarity. As can be seen, there is a reasonable match between both models, showing some differences at the tails. In Figure $4 \mathrm{~b}$, in a similar way the masking curves are shown but now resulting from a complex spectrum (part of a harpsichord signal). It can be seen that the masking curves differ systematically showing much smoother masking curves for the spectral integration model as compared to the MPEG model. For the spectral integration model masking curves are considerably higher in spectral valleys. This effect is a direct consequence of the spectral integration assumption that was adopted in our model (cf. (6)). In the spectral valleys of the masker, distortion signals can only be detected using the auditory filter centred on the distortion which will lead to relatively high masked thresholds. This is so because off-frequency filters will be dominated by the masker spectrum. However, detection of distortion signals at the spectral peaks of the masker is mediated by a range of auditory filters centred around the peak, resulting in relatively low masked thresholds. In this case the off-frequency filters will reveal similar distortion-to-masker ratios as the on-frequency filter. Thus, in the model proposed here, detection differences between peaks and troughs are smaller, resulting in smoother masking curves as compared to those observed in a spreading-based model such as the ISO MPEG model.
The smoothening effect is observed systematically in complex signal spectra typically encountered in practical situations and represents the main difference between the spectral integration model presented here and existing spreadingbased models.

\section{APPLICATION TO SINUSOIDAL MODELLING}

Sinusoidal modelling has proven to be an efficient technique for the purpose of coding speech signals [40]. More recently, it has been shown that this method can also be exploited for low-rate audio coding, for example, [41, 42, 43]. To account for the time-varying nature of the signal, the sinusoidal analysis/synthesis is done on a segment-by-segment basis, with each segment being modelled as a sum of sinusoids. The sinusoidal parameters have been selected with a number of methods, including spectral peak-picking [44], analysis-bysynthesis [41, 43], and subspace-based methods [42].

In this section we describe an algorithm for selecting sinusoidal components using the psychoacoustical model described in the previous section. The algorithm is based on the matching pursuit algorithm [45], a particular analysisby-synthesis method. Matching pursuit approximates a signal by a finite expansion into elements (functions) chosen from a redundant dictionary. In the example of sinusoidal modelling, one can think of such functions as (complex) exponentials or as real sinusoidal functions. Matching pursuit is a greedy, iterative algorithm which searches the dictionary for the function that best matches the signal and subtracts this function (properly scaled) to form a residual signal to be approximated in the next iteration.

In order to determine which is the best matching function or dictionary element at each iteration, we need to formalise the problem. To do so, let $\mathscr{D}=\left(g_{\xi}\right)_{\xi \in \Gamma}$ be a complete dictionary, that is, a set of elements indexed by $\xi \in \Gamma$, where $\Gamma$ is an arbitrary index set. As an example, consider a dictionary consisting of complex exponentials $g_{\xi}=e^{i 2 \pi \xi(\cdot)}$. In this case, the index set $\Gamma$ is given by $\Gamma=[0,1)$. Obviously, the indexing parameter $\xi$ is nothing more than the frequency of the complex exponential. Given a dictionary $\mathcal{D}$, the best matching function can be found by, for each and every function, computing the best approximation and selecting that function whose corresponding approximation is "closest" to the original signal.

In order to facilitate the following discussion, we assume without loss of generality that $\left\|g_{\xi}\right\|=1$ for all $\xi$. Given a particular function $g_{\xi}$, the best possible approximation of the signal $x$ is obtained by the orthogonal projection of $x$ onto the subspace spanned by $g_{\xi}$ (see Figure 5). This projection is given by $\left\langle x, g_{\xi}\right\rangle g_{\xi}$. Hence, we can decompose $x$ as

$$
x=\left\langle x, g_{\xi}\right\rangle g_{\xi}+R x,
$$

where $R x$ is the residual signal after subtracting the projection $\left\langle x, g_{\xi}\right\rangle g_{\xi}$. The orthogonality of $R x$ and $g_{\xi}$ implies that

$$
\|x\|^{2}=\left|\left\langle x, g_{\xi}\right\rangle\right|^{2}+\|R x\|^{2} .
$$




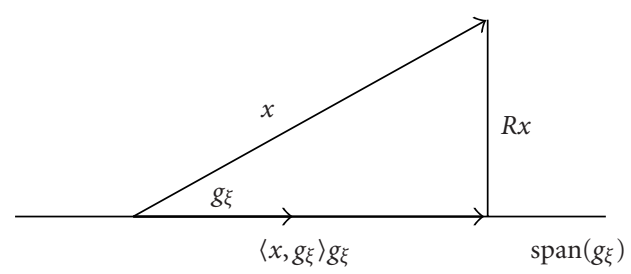

FIGURE 5: Orthogonal projection of $x$ onto $\operatorname{span}\left(g_{\xi}\right)$.

We can do this decomposition for each and every dictionary element and the best matching one is then found by selecting the element $g_{\xi}$, for which $\|R x\|$ is minimal, or, equivalently, for which $\left|\left\langle x, g_{\xi}\right\rangle\right|$ is maximal. A precise mathematical formulation of this phrase is

$$
\xi^{\prime}=\arg \sup _{\xi \in \Gamma}\left|\left\langle x, g_{\xi}\right\rangle\right|
$$

It must be noted that the matching pursuit algorithm is only optimal for a particular iteration. If we subtract the approximation to form a residual signal and approximate this residual in a similar way as we approximated the original signal, then the two dictionary elements thus obtained are not jointly optimal; it is in general possible to find two different elements which together form a better approximation. This is a direct consequence of the greedy nature of the algorithm. The two dictionary elements which together are optimal could be obtained by projecting the signal $x$ onto all possible two-dimensional subspaces. This, however, is in general very computationally complex. An alternative solution to this problem is to apply, after each iteration, a Newton optimisation step [46].

To account for human auditory perception, the unitnorm dictionary elements can be scaled [43], which is equivalent to scaling the inner products in (16). We will refer to this method as the weighted matching pursuit (WMP) algorithm. While this method performs well, it can be shown that it does not provide a consistent selection measure for elements of finite time support [47]. Rather than scaling the dictionary elements, we introduce a matching pursuit algorithm where psychoacoustical properties are accounted for by a norm on the signal space. We will refer to this method as psychoacoustical matching pursuit (PAMP). As mentioned in Section 3 (see (11)), the perceptual distortion can be expressed as

$$
D=\sum_{f} \frac{|\widehat{\varepsilon}(f)|^{2}}{\hat{v}^{2}(f)}=\sum_{f} \hat{a}(f)|\widehat{\varepsilon}(f)|^{2},
$$

where $\hat{a}=\hat{v}^{-2}$. It follows from (10) that

$$
\hat{a}(f)=C_{s} L_{\text {eff }} \sum_{i} \frac{\left|\hat{h}_{\mathrm{om}}(f)\right|^{2}\left|\hat{\gamma}_{i}(f)\right|^{2}}{N M_{i}+C_{a}} .
$$

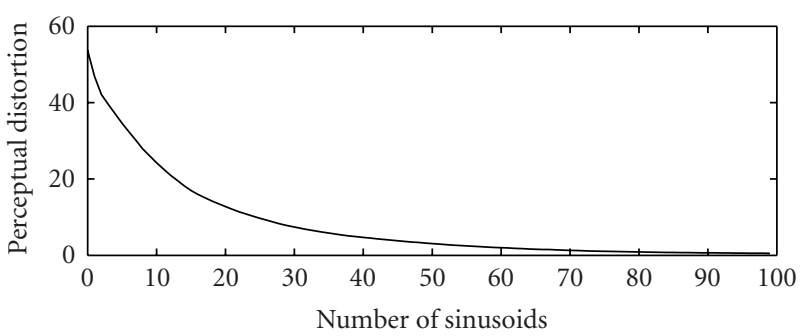

FIGURE 6: Perceptual distortion associated with the residual signal after sinusoidal modelling as a function of the number of sinusoidal components that were extracted.

By inspection of (18), we conclude that $\hat{a}$ is real and positive so that, in fact, the perceptual distortion measure (17) defines a norm

$$
\|x\|^{2}=\sum_{f} \hat{a}(f)|\hat{x}(f)|^{2}
$$

This norm is induced by the inner product

$$
\langle x, y\rangle=\sum_{f} \hat{a}(f) \hat{x}(f) \hat{y}^{*}(f),
$$

facilitating the use of the distortion measure in selecting the perceptually best matching dictionary element in a matching pursuit algorithm. In Figure 6, the perceptual distortion associated with the residual signal is shown as a function of the number of real-valued sinusoids that have been extracted for a short segment of a harpsichord excerpt (cf. (11)). As can be seen the perceptually most relevant components are selected first, resulting in a fast reduction of the perceptual distortion for the first components. For a detailed description the reader is referred to $[47,48]$. The fact that the distortion detectability defines a norm on the underlying signal space is important, since it allows for incorporating psychoacoustics in optimisation algorithms. Indeed, rather than minimising the commonly used $l_{2}$-norm, we can minimise the perceptually relevant norm given by (19). Examples include ratedistortion optimisation [32], linear predictive coding [49], and subspace-based modelling techniques [50].

\section{COMPARISON WITH THE ISO MPEG MODEL IN A LISTENING TEST}

In this section we assess the performance of the proposed perceptual model in the context of sinusoidal parameter estimation. The PAMP method for estimating perceptually relevant sinusoids relies on the weighting function $\hat{a}$ which, by definition, is the inverse of the masking curve. Equation (18) describes how to compute the masking curve for the proposed perceptual model. We compare the use of the proposed perceptual model in PAMP to the situation where the masking curve is computed using the MPEG-1 Layer I-II (ISO/IEC 11172-3) psychoacoustical model [1]. There are several reasons for comparison with the MPEG psychoacoustic model; the model provides a well-known 
reference and because of its frequent application, it is still a de facto state-of-the-art model.

Using the MPEG-1 psychoacoustic model masking curve directly in the PAMP algorithm for sinusoidal extraction is not reasonable because the MPEG-1 psychoacoustic model was developed to predict the masking curve in the case of noise maskees (distortion signals). It predicts for every frequency bin how much distortion can be added within the critical band centred around the frequency bin. This prediction is, however, too conservative in the case that distortions are sinusoidal in nature since in this case the distortion energy is not spread over a complete critical band but is concentrated in one frequency bin only. Hence, we can adapt the MPEG-1 model by scaling the masking function with the critical bandwidth such that the model now predicts the detection thresholds in the case of sinusoidal distortion. The net effect of this compensation procedure is an increase of the masking curve at high frequencies by about $10 \mathrm{~dB}$, thereby de-emphasizing high-frequency regions during sinusoidal estimation. In fact, this masking power increase at higher frequencies reduces the gap between the masking curves between the ISO MPEG model and the proposed model (cf. Figure 4) By applying this modification to the ISO MPEG model, and by extending the FFT order to the size of the PAMP dictionary, it is suited to be used in the PAMP method. The dictionary elements in our implementation of the PAMP method were real-valued sinusoidal functions windowed with a Hanning window, identical to the window used in the analysis-synthesis procedure described below.

In the following, we present results obtained by listening tests with audio signals. The signals are mono, sampled at $44.1 \mathrm{kHz}$, where each sample is represented by 16 bits. The test excerpts are Carl Orff, Castanet, Céline Dion, Harpsichord Solo, contemporary pop music, and Suzanne Vega.

The excerpts were segmented into fixed-length frames of 1024 samples (corresponding to 23.2 milliseconds) with an overlap of $50 \%$ between consecutive frames using a Hanning window. For each signal frame, a fixed number of perceptually relevant sinusoids per frame were extracted using the PAMP method described above, where the perceptual weighting functions $\hat{a}$ were generated from masking curve derived from the proposed perceptual model (see (18)) and the modified MPEG model described above, respectively. For the MPEG model we made use of the recommendations of MPEG Layer II, since these support input frame lengths of 1024 samples. The masking curves were calculated from the Hanning-windowed original signal contained within the same frame that is being modelled using the PAMP method. Finally, modelled frames were synthesized from the estimated sinusoidal parameters and concatenated to form modelled test excerpts, using a Hanning window-based overlapadd procedure.

To evaluate the performance of the proposed method, we used a subjective listening test procedure which is somewhat comparable to the MUSHRA test (multistimulus test with hidden reference and anchors) [51]. For each test excerpt, listeners were asked to rank 6 different versions: 4 excerpts modelled using the modified MPEG masking curve and fixed
TABLE 1: Scores used in subjective test.

\begin{tabular}{cc}
\hline Score & Equivalent \\
\hline 5 & Best \\
4 & Good \\
3 & Medium \\
2 & Poor \\
1 & Poorest \\
\hline
\end{tabular}

model orders (i.e., the number of sinusoidal components per segment) of $K=20,25,30$, and $K=35$, and one excerpt modelled using the proposed perceptual model with $K=25$. In addition, to have a low-quality reference signal, an excerpt modelled with $K=30$, but using the unmodified MPEG masking curve was included. As a reference, the listeners had the original excerpt available as well, which was identified to the subjects. Unlike the MUSHRA test, no hidden reference and no anchors were presented to the listeners.

The test excerpts were presented in a "parallel" way, using the interactive benchmarking tool described in [52] as an interface to the listeners. For each excerpt, listeners were requested to rank the different modelled signals on a scale from 1-5 (in steps of 0.1 ) as outlined in Table 1. The listeners were instructed to use the complete scale such that the poorest-quality excerpt was rated with 1 and the highestquality excerpt with 5 . The excerpts were presented through high-quality headphones (Beyer-Dynamic DT990 PRO) in a quiet room, and the listeners could listen to each signal version as often as needed to determine the ranking. A total of 12 listeners participated in the listening test, of which 6 listeners worked in the area of acoustic signal processing and had previously participated in such tests. The authors did not participate in the test.

Figure 7 shows the overall scores of the listening test, averaged across all listeners and excerpts. The circles represent the median score, and the error bars depict 25 and 75 percent ranges of the total response distributions. As can be seen, the excerpts generated with the proposed perceptual model (SiCAS@25) show better average subjective performance than any of the excerpts based on the MPEG psychoacoustic model, except for the MPEG case using a fixed model order of 35 (MPEG@35). As expected, the MPEG-based excerpts have decreasing quality scores for decreasing model order. Furthermore, the low-quality anchor (MPEG@30nt, i.e., the MPEG model without spectral tilt modification) received the lowest-quality score on average. The statistical difference between the quality scores was analysed using a paired t-test using a significance level of $p<0.01$, and by working on the score differences between the proposed perceptual model and each of the MPEG-based methods. The $H_{0}$ hypothesis was that the mean of such difference distribution was zero $\left(\mu_{\Delta}=0\right)$, while the alternative hypothesis $H_{1}$ was that $\mu_{\Delta}>0$. The statistical analysis supports the quality ordering suggested by Figure 7. In particular, there is a statistically significant improvement in using the proposed perceptual model (SiCAS@25) over any of the MPEG-based methods except for MPEG@35 which performs better than SiCAS@25 $\left(p<7.0 \cdot 10^{-3}\right)$. In fact, the model presented here 


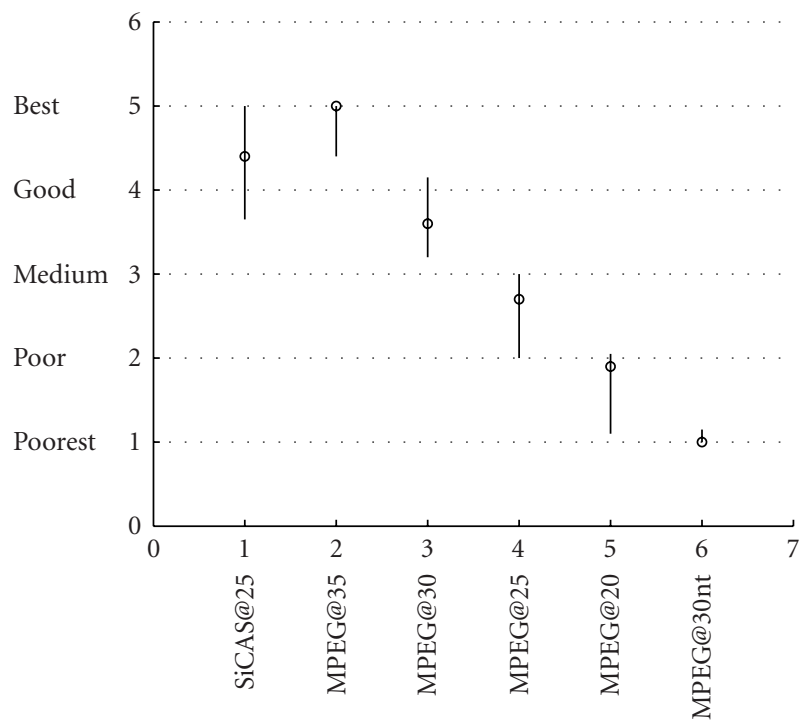

FIGURE 7: Subjective test results averaged across all listeners and excerpts.

leads to a reduction of more than $20 \%$ in terms of number of sinusoids needed to represent signals at a given quality level.

As mentioned already in Section 5 the most relevant difference between the proposed model and the ISO MPEG model is the incorporation of spectral integration properties in the proposed model. This leads to systematically smoother masking curves such as predicted by our model for complex masker spectra (cf. Figure 4). The effect of this is that fewer sinusoidal components are used for modelling spectral valleys of a signal with the proposed perceptual model as compared to the ISO MPEG model. We think that this difference accounts for the improvement in modelling efficiency that we observed in the listening tests and we expect that similar improvements would have been observed when our approach was compared to other perceptual models that are based on the spectral-spreading approach such as those used in the ISO MPEG model.

\section{CONCLUSIONS}

In this paper we presented a psychoacoustical model that is suited for predicting masked thresholds for sinusoidal distortions. The model relies on signal detection theory and incorporates more recent insights about spectral and temporal integration in auditory masking. We showed that, as a consequence, the model is able to predict distortion detectabilities. In fact, the distortion detectability defines a (perceptually relevant) norm on the underlying signal space which is beneficial for optimisation algorithms such as rate-distortion optimisation or linear predictive coding. The model proves to be very suitable for application in the context of sinusoidal modelling, although it is also applicable in other audio coding contexts such as transform coding. A comparative listening test using a sinusoidal analysis method called psychoacoustical matching pursuit showed a clear preference for the model presented here over the ISO MPEG model [1].
More specifically, the model presented here leads to a reduction of more than $20 \%$ in terms of number of sinusoids needed to represent signals at a given quality level.

\section{ACKNOWLEDGMENTS}

The authors would like to thank Nicolle H. van Schijndel, Gerard Hotho, and Jeroen Breebaart and the reviewers for their helpful comments on this manuscript. Furthermore, the authors thank the participants in the listening test. The research was supported by Philips Research, the Technology Foundation STW, Applied Science Division of NWO, the Technology Programme of the Dutch Ministry of Economic Affairs, and the EU project ARDOR, IST-2001-34095.

\section{REFERENCES}

[1] IISO/MPEG Committee, Coding of moving pictures and associated audio for digital storage media at up to about $1.5 \mathrm{Mbit} / \mathrm{s}$ - part 3: Audio, 1993, ISO/IEC 11172-3.

[2] T. Yoshida, "The rewritable minidisc system," Proc. IEEE, vol. 82, no. 10, pp. 1492-1500, 1994.

[3] A. Hoogendoorn, "Digital compact cassette," Proc. IEEE, vol. 82, no. 10, pp. 1479-1589, 1994.

[4] T. Painter and A. Spanias, "Perceptual coding of digital audio," Proc. IEEE, vol. 88, no. 4, pp. 451-515, 2000.

[5] K. N. Hamdy, M. Ali, and A. H. Tewfik, "Low bit rate high quality audio coding with combined harmonic and wavelet representation," in Proc. IEEE Int. Conf. Acoustics, Speech, Signal Processing (ICASSP '96), vol. 2, pp. 1045-1048, Atlanta, Ga, USA, May 1996.

[6] S. N. Levine, Audio representations for data compression and compressed domain processing, Ph.D. thesis, Stanford University, Stanford, Calif, USA, 1998.

[7] H. Purnhagen and N. Meine, "HILN-the MPEG-4 parametric audio coding tools," in Proc. IEEE Int. Symp. Circuits and Systems (ISCAS '00), vol. 2000, pp. 201-204, Geneva, Switzerland, May 2000.

[8] W. Oomen, E. Schuijers, B. den Brinker, and J. Breebaart, "Advances in parametric coding for high-quality audio," in Proc. 114th AES Convention, Amsterdam, The Netherlands, March 2003, preprint 5852.

[9] F. P. Myburg, Design of a scalable parametric audio coder, Ph.D. thesis, Technische Universiteit Eindhoven, Eindhoven, The Netherlands, 2004.

[10] H. S. Malvar, Signal Processing with Lapped Transforms, Artech House, Boston, Mass, USA, 1992.

[11] P. P. Vaidyanathan, Multirate Systems and Filter Banks, Prentice Hall Signal Processing Series, Prentice Hall, Englewood Cliffs, NJ, USA, 1993.

[12] J. E. Hawkins and S. S. Stevens, "The masking of pure tones and of speech by white noise," Journal of the Acoustical Society of America, vol. 22, pp. 6-13, 1950.

[13] T. S. Verma, A perceptually based audio signal model with application to scalable audio coding, Ph.D. thesis, Stanford University, Stanford, Claif, USA, 1999.

[14] R. L. Wegel and C. E. Lane, "The auditory masking of one pure tone by another and its probable relation to the dynamics of the inner ear," Phys. Rev., vol. 23, pp. 266-285, 1924.

[15] H. Fletcher, "Auditory patterns," Reviews of Modern Physics, vol. 12, no. 1, pp. 47-65, 1940.

[16] P. M. Sellick, R. Patuzzi, and B. M. Johnstone, "Measurements of BM motion in the guinea pig using Mössbauer technique," Journal of the Acoustical Society of America, vol. 72, pp. 131141, 1982. 
[17] J. P. Egan and H. W. Hake, "On the masking pattern of a simple auditory stimulus," Journal of the Acoustical Society of America, vol. 22, pp. 622-630, 1950.

[18] K. G. Yates, I. M. Winter, and D. Robertson, "Basilar membrane nonlinearity determines auditory nerve rate-intensity functions and cochlear dynamic range," Hearing Research, vol. 45, no. 3, pp. 203-220, 1990.

[19] R. D. Patterson, "Auditory filtershapes derived with noise stimuli," Journal of the Acoustical Society of America, vol. 59, pp. 1940-1947, 1976.

[20] M. van der Heijden and A. Kohlrausch, "The role of envelope fluctuations in spectral masking," Journal of the Acoustical Society of America, vol. 97, no. 3, pp. 1800-1807, 1995.

[21] M. van der Heijden and A. Kohlrausch, "The role of distortion products in masking by single bands of noise," Journal of the Acoustical Society of America, vol. 98, no. 6, pp. 3125-3134, 1995.

[22] J. P. Egan, W. A. Lindner, and D. McFadden, "Masking-level differences and the form of the psychometric function," Perception and Psychophysics, vol. 6, pp. 209-215, 1969.

[23] D. M. Green and J. A. Swets, Signal Detection Theory and Psychophysics, Krieger, New York, NY, USA, 1974.

[24] S. Buus, E. Schorer, M. Florentine, and E. Zwicker, "Decision rules in detection of simple and complex tones," Journal of the Acoustical Society of America, vol. 80, no. 6, pp. 1646-1657, 1986.

[25] A. Langhans and A. Kohlrausch, "Spectral integration of broadband signals in diotic and dichotic masking experiments," Journal of the Acoustical Society of America, vol. 91, no. 1, pp. 317-326, 1992.

[26] S. van de Par, A. Kohlrausch, J. Breebaart, and M. McKinney, "Discrimination of different temporal envelope structures of diotoic and dichotic targets signals within diotic wide-band noise," in Proc. 13th International Symposium on Hearing, pp. 334-340, Dourdan, France, August 2003.

[27] G. van den Brink, "Detection of tone pulse of various durations in noise of various bandwidths," Journal of the Acoustical Society of America, vol. 36, pp. 1206-1211, 1964.

[28] S. van de Par and A. Kohlrausch, "Application of a spectrally integrating auditory filterbank model to audio coding," in Fortschritte der Akustik, Plenarvorträge der 28. Deutschen Jahrestagung für Akustik, DAGA-02, pp. 484-485, Bochum, Germany, 2002.

[29] T. Dau, D. Püschel, and A. Kohlrausch, "A quantitative model of the 'effective' signal processing in the auditory system. I. Model structure," Journal of the Acoustical Society of America, vol. 99, no. 6, pp. 3615-3622, 1996.

[30] R. D. Patterson, "The sound of a sinusoid; spectral models," Journal of the Acoustical Society of America, vol. 96, no. 3, pp. 1409-1418, 1994.

[31] B. R. Glasberg and B. C. J. Moore, "Derivation of auditory filter shapes from notched-noise data," Hearing Research, vol. 47, no. 1-2, pp. 103-138, 1990.

[32] R. Heusdens, J. Jensen, W. B. Kleijn, V. Kot, O. Niamut, S. van de Par, N. H. van Schijndel, and R. Vafin, "Sinusoidal coding of audio and speech," in preparation for Journal of the Audio Engineering Society, 2005.

[33] B. C. J. Moore, An Introduction to the Psychology of Hearing, Academic Press, London, UK, 3rd edition, 1989.

[34] G. Charestan, R. Heusdens, and S. van de Par, "A gammatone based psychoacoustical modeling approach for speech and audio coding," in Proc. ProRISC/IEEE: Workshop on Circuits, Systems and Signal Processing, pp. 321-326, Veldhoven, The Netherlands, November 2001.

[35] A. J. M. Houtsma, "Hawkins and Stevens revisited at low frequencies," Journal of the Acoustical Society of America, vol. 103 , no. 5, pp. 2848-2848, 1998.

[36] E. Zwicker and A. Jaroszewski, "Inverse frequency dependence of simultaneous tone-on-tone masking patterns at low levels," Journal of the Acoustical Society of America, vol. 71, pp. 1508$1512,1982$.

[37] A. Langhans and A. Kohlrausch, "Differences in auditory performance between monaural and diotic conditions. I. Masked thresholds in frozen noise," Journal of the Acoustical Society of America, vol. 91, pp. 3456-3470, 1992.

[38] S. van de Par and A. Kohlrausch, "Dependence of binaural masking level differences on center frequency, masker bandwidth and interaural parameters," Journal of the Acoustical Society of America, vol. 106, pp. 1940-1947, 1999.

[39] E. Zwicker and H. Fastl, Psychoacoustics_Facts and Models, Springer, Berlin, Germany, 2nd edition, 1999.

[40] R. J. McAulay and T. F. Quatieri, "Sinusoidal coding," in Speech Coding and Syntesis, W. B. Kleijn and K. K. Paliwal, Eds., chapter 4, pp. 121-173, Elsevier Science B. V., Amsterdam, The Netherlands, 1995.

[41] M. Goodwin, "Matching pursuit with damped sinusoids," in Proc. IEEE Int. Conf. Acoustics, Speech, Signal Processing (ICASSP '97), vol. 3, pp. 2037-2040, Munich, Germany, April 1997.

[42] J. Nieuwenhuijse, R. Heusdens, and E. F. Deprettere, "Robust exponential modeling of audio signals," in Proc. IEEE Int. Conf. Acoustics, Speech, Signal Processing (ICASSP '98), vol. 6, pp. 3581-3584, Seattle, Wash, USA, May 1998.

[43] T. S. Verma and T. H. Y. Meng, "Sinusoidal modeling using frame-based perceptually weighted matching pursuits," in Proc. IEEE Int. Conf. Acoustics, Speech, Signal Processing (ICASSP '99), vol. 2, pp. 981-984, Phoenix, Ariz, USA, May 1999.

[44] R. J. McAulay and T. F. Quatieri, "Speech analysis/synthesis based on a sinusoidal representation," IEEE Trans. Acoust., Speech, Signal Processing, vol. 34, no. 4, pp. 744-754, 1986.

[45] S. G. Mallat and Z. Zhang, "Matching pursuits with timefrequency dictionaries," IEEE Trans. Signal Processing, vol. 41, no. 12 , pp. 3397-3415, 1993.

[46] K. Vos and R. Heusdens, "Rate-distortion optimal exponential modeling of audio and speech signals," in Proc. 21st Symposium on Information Theory in the Benelux, pp. 77-84, Wassenaar, The Netherlands, May 2000.

[47] R. Heusdens, R. Vafin, and W. B. Kleijn, "Sinusoidal modeling using psychoacoustic-adaptive matching pursuits," IEEE Signal Processing Lett., vol. 9, no. 8, pp. 262-265, 2000.

[48] R. Heusdens and S. van de Par, "Rate-distortion optimal sinusoidal modeling of audio and speech using psychoacoustical matching pursuits," in Proc. IEEE Int. Conf. Acoustics, Speech, Signal Processing (ICASSP '02), vol. 2, pp. 1809-1812, Orlando, Fla, USA, May 2002.

[49] R. C. Hendriks, R. Heusdens, and J. Jensen, "Perceptual linear predictive noise modelling for sinusoid-plus-noise audio coding," in Proc. IEEE Int. Conf. Acoustics, Speech, Signal Processing (ICASSP '04), vol. 4, pp. 189-192, Montreal, Quebec, Canada, May 2004.

[50] J. Jensen, R. Heusdens, and S. H. Jensen, "A perceptual subspace approach for modeling of speech and audio," IEEE Trans. Speech Audio Processing, vol. 12, no. 2, pp. 121-132, 2004.

[51] ITU, ITU-R BS 1534. Method for subjective assessment of intermediate quality level of coding systems, 2001.

[52] O. A. Niamut, Audio codec Benchmark manual, Department of Mediamatics, Delft University of Technology, January 2003. 
Steven van de Par studied physics at the Eindhoven University of Technology (TU/e), and received his Ph.D. degree in 1998 from the Institute for Perception Research on a topic related to binaural hearing. As a Postdoctoral Researcher at the same institute, he studied auditory-visual interaction and he was a Guest Researcher at the University of Connecticut Health Centre. In the beginning of 2000 he joined Philips Research, Eindhoven. Main fields of expertise are auditory and multisensory perception and low-bit-rate audio coding. He published various papers on binaural detection, auditory-visual synchrony perception, and audio-coding-related topics. He participated in several projects on low-bit-rate audio coding based on sinusoidal techniques and is presently participating in the EU Adaptive Rate-Distortion Optimized Audio codeR (ARDOR) project.

Armin Kohlrausch studied physics at the University of Göttingen, Germany, and specialized in acoustics. He received his M.S. degree in 1980 and his Ph.D. degree in 1984, both in perceptual aspects of sound. From 1985 until 1990 he worked at the Third Physical Institute, University of Göttingen, and was responsible for research and teaching in the fields psychoacoustics and room acoustics. In 1991 he joined the Philips Re-

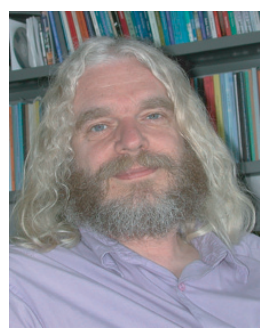
search Laboratories, Eindhoven, and worked in the Speech and Hearing Group, Institute for Perception Research (IPO). Since 1998, he has combined his work at Philips Research Laboratories with a Professor position for multisensory perception at the TU/e. In 2004 he was appointed a Research Fellow of Philips Research. $\mathrm{He}$ is a member of a great number of scientific societies, both in Europe and the USA. Since 1998 he has been a Fellow of the Acoustical Society of America and serves currently as an Associate Editor for the Journal of the Acoustical Society of America, covering the areas of binaural and spatial hearing. His main scientific interests are in the experimental study and modelling of auditory and multisensory perception in humans and the transfer of this knowledge to industrial media applications.

Richard Heusdens is an Associate Professor in the Department of Mediamatics, Delft University of Technology. He received his M.S. and Ph.D. degrees from the Delft University of Technology, the Netherlands, in 1992 and 1997, respectively. In the spring of 1992 he joined the Digital Signal Processing Group, Philips Research Laboratories, Eindhoven, the Netherlands. He has worked on various topics in the field of signal process-

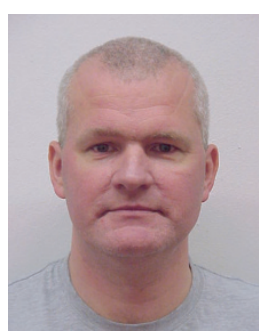
ing, such as image/video compression and VLSI architectures for image-processing algorithms. In 1997, he joined the Circuits and Systems Group, Delft University of Technology, where he was a Postdoctoral Researcher. In 2000, he moved to the Information and Communication Theory (ICT) Group where he became an Assistant Professor, responsible for the audio and speech processing activities within the ICT Group. Since 2002, he has been an Associate Professor. Research projects he is involved in cover subjects such as audio and speech coding, speech enhancement, and digital watermarking of audio.
Jesper Jensen received the M.S. and Ph.D. degrees from Aalborg University, Aalborg, Denmark, in 1996 and 2000, respectively, both in electrical engineering. From 1996 to 2001, he was with the Center for PersonKommunikation (CPK), Aalborg University, as a Researcher, Ph.D. student, and Assistant Research Professor. In 1999, he was a Visiting Researcher at the Center for Spoken Language Research, University of

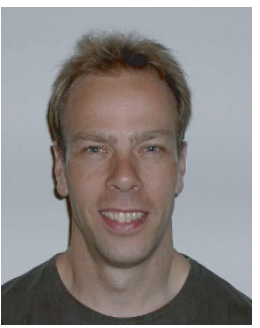
Colorado at Boulder. Currently, he is a Postdoctoral Researcher at Delft University of Technology, Delft, the Netherlands. His main research interests are in digital speech and audio signal processing, including coding, synthesis, and enhancement.

Søren Holdt Jensen received the M.S. degree in electrical engineering from Aalborg University, Denmark, in 1988, and the Ph.D. degree from the Technical University of Denmark, in 1995. He has been with the Telecommunications Laboratory of Telecom Denmark, the Electronics Institute of the Technical University of Denmark, the Scientific Computing Group of the Danish Computing Center for Research and Educa-

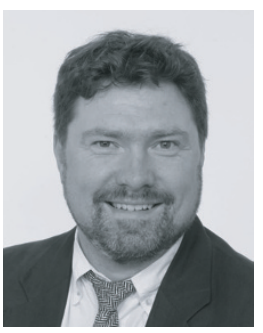
tion (UNI-C), the Electrical Engineering Department of Katholieke Universiteit Leuven, Belgium, the Center for PersonKommunikation (CPK) of Aalborg University, and is currently an Associate Professor in the Department of Communication Technology, Aalborg University. His research activities are in digital signal processing, communication signal processing, and speech and audio processing. He is a Member of the Editorial Board of EURASIP Journal on Applied Signal Processing, and a former Chairman of the IEEE Denmark Section and the IEEE Denmark Section's Signal Processing Chapter. 